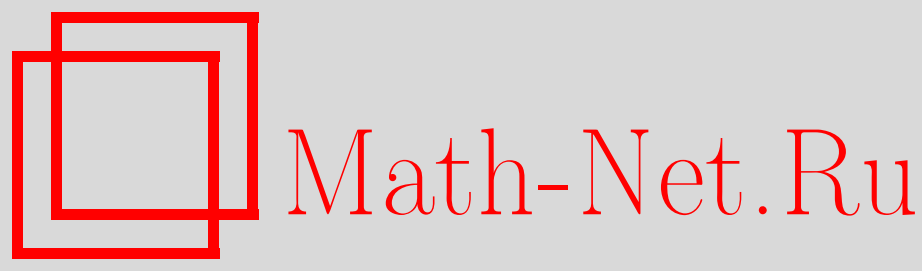

С. А. Соколовский, K кинетике полярона в методе сокращенного описания Боголюбова, ТМ $\Phi, 2011$, том 168, номер 2, 341-357

DOI: https://doi.org/10.4213/tmf6684

Использование Общероссийского математического портала Math-Net.Ru подразумевает, что вы прочитали и согласны с пользовательским соглашением http://www.mathnet.ru/rus/agreement

Параметры загрузки:

IP : 54.80 .73 .141

26 апреля 2023 г., 18:08:32

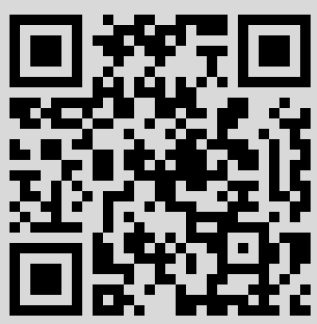




\title{
К КИНЕТИКЕ ПОЛЯРОНА В МЕТОДЕ СОКРАЩЕННОГО ОПИСАНИЯ БОГОЛЮБОВА
}

\begin{abstract}
В рамках метода сокращенного описания неравновесных состояний Боголюбова, основанного на его функциональной гипотезе, получено кинетическое уравнение для произвольных неоднородных состояний электрона в полярном кристалле при наличии сильного электрического поля. В пространственно-однородном случае при наличии слабого электрического поля на основе метода сокращенного описания исследовано выравнивание скоростей и температур поляронного газа малой плотности и фононной подсистемы. Установлено, что неравновесная функция распределения полярона отличается от распределения Максвелла уже в линейном приближении по малым разностям скоростей и температур подсистем. Вычислены соответствующие времена релаксации и подвижность полярона.
\end{abstract}

Ключевые слова: полярон, метод сокращенного описания Боголюбова, функциональная гипотеза, кинетическое уравнение, сильно неоднородные состояния, сильное электрическое поле, релаксационные явления, подвижность полярона.

\section{1. ВВЕДЕНИЕ}

Настоящая работа посвящена исследованию кинетики электрон-фононной системы полярного кристалла с помощью метода сокращенного описания Боголюбова, основанного на его функциональной гипотезе [1]. Весомый вклад в изучение этой системы внесли Боголюбов и Боголюбов (мл.), результаты которых суммированы в монографии [2]. В первую очередь речь идет о теории полярона, т. е. об одном электроне, взаимодействующем с фононным полем [3]. В основу рассмотрения положена модель Фрёлиха взаимодействия электрона с фононами (см., например, работы [4], [5]). Следует отметить, что Боголюбов в своей теории полярона не использовал метод сокращенного описания [2], а исходил из первых принципов статистической механики и только в итоговых формулах считал взаимодействие слабым. Хотя в настоящей работе функциональная гипотеза не доказывается, мы полагаем, что соответствующее сокращенное описание реализуется в действительности, если вводимые этой гипотезой математические объекты могут быть вычислены в рамках некоторой теории возмущений и вклады этой теории сходятся. Заметим в этой

* Приднепровская государственная академия строительства и архитектуры, Днепропетровск, Украина. E-mail: sersokol@list.ru 
связи, что подходы к доказательству функциональной гипотезы обсуждались в литературе (см., например, работы [6], [7]).

В используемой нами модели Фрёлиха взаимодействие электрона с ионами описывается с помощью кулоновского потенциала, поэтому спин электрона не учитывается. При отсутствии колебаний ионов электрон движется в периодическом поле решетки. В рамках рассматриваемой модели мы, как и другие авторы (см., например, монографию [2]), не учитываем модификацию спектра электрона в этом поле и изучаем электрон со спектром $\varepsilon_{p} \equiv p^{2} / 2 m$ ( $m$ - эффективная масса электрона), который взаимодействует с фононным полем. Учет этого взаимодействия электрона сводится к его взаимодействию с продольными оптическими фононами [4], [5]. Область изменения волновых векторов фононов для простоты, как и во многих работах (см., например, [2], [8]), мы не ограничиваем зоной Бриллюэна. Взаимодействием между фононами, которое не сохраняет их число, также пренебрегаем. Фононная подсистема (фононный газ) рассматривается нами как покоящаяся равновесная система. Сначала мы изучаем кинетику одного электрона, поэтому использование для его описания вторичного квантования нецелесообразно, и оператор Гамильтона системы выбирается в виде

$$
\begin{gathered}
\widehat{H}=\widehat{H}_{s}+\widehat{H}_{b}+\widehat{H}_{s b}, \quad \widehat{H}_{s}=\varepsilon_{\hat{p}}+e \hat{x}_{n} E_{n}, \quad \widehat{H}_{b}=\sum_{k} \hbar \omega_{k} c_{k}^{+} c_{k}, \\
\widehat{H}_{s b}=\frac{1}{\sqrt{V}} \sum_{k}\left(\widehat{A}_{k}^{+} c_{k}+\widehat{A}_{k} c_{k}^{+}\right), \quad \widehat{A}_{k} \equiv-i g_{k} e^{-i k_{n} \hat{x}_{n}},
\end{gathered}
$$

где $c_{k}, c_{k}^{+}$- операторы уничтожения и рождения фононов, $\hat{x}_{n}, \hat{p}_{n}$ - операторы координаты и импульса электрона, $e$ - абсолютная величина заряда электрона, $V$ объем системы. В модели Фрёлиха частота фононов $\omega_{k}$ и функция $g_{k}$, определяющая интенсивность взаимодействия в системе, обычно конкретизируются следующим образом:

$$
\omega_{k}=\omega_{0}, \quad g_{k}=2 \frac{\hbar \omega_{0}}{k}\left(\frac{\pi \alpha}{k_{0}}\right)^{1 / 2}, \quad k_{0} \equiv\left(\frac{2 m \omega_{0}}{\hbar}\right)^{1 / 2},
$$

где $\alpha$ - безразмерная постоянная Фрёлиха, $k_{0}$ - волновое число электрона, при котором его энергия совпадает с энергией фонона, $\omega_{0}$ - частота продольных фононов (см., например, монографию [5]). Нами в (1) и далее используются периодические граничные условия $\left(k_{l}=2 \pi n_{l} / V^{1 / 3}, n_{l} \in \mathbb{Z}\right)$. Именно в такой формулировке модель полярона изучалась в книге [2], статье [8] и работах других авторов, отмечавших важное теоретическое значение ее исследования.

План настоящей работы следующий. В разделе 2 на основе функциональной гипотезы в приближении слабой связи выводится кинетическое уравнение для произвольных пространственно-неоднородных состояний электрона при наличии сильного однородного электрического поля $E_{n}$ (случай отсутствия поля в рамках аналогичного подхода рассмотрен в нашей работе [9]). В пространственно-однородном случае при наличии слабого электрического поля наше рассмотрение приводит к кинетическому уравнению типа Больцмана, выведенному Боголюбовым методом исключения бозонных переменных (см. ссылки в книге [2]).

В разделе 3 на основе данного уравнения изучается релаксация (выравнивание) температур и скоростей в пространственно-однородной электрон-фононной системе, 
состоящей из поляронного газа малой плотности и равновесной фононной подсистемы. Это возможно, поскольку кинетическое уравнение для такого газа не отличается от упомянутого уравнения. Таким образом, речь идет о релаксации температур и скоростей в двухкомпонентной системе. В основу рассмотрения нами положено обобщение метода Чепмена-Энскога, которое вытекает из его понимания как метода сокращенного описания Боголюбова, примененного для решения кинетических уравнений (см. стандартное изложение метода в книгах [6], [10]). Идея такого отношения к методу Чепмена-Энскога была предложена самим Боголюбовым в работе [1] (см. также [6]). На этой основе нами развивается приближенный подход к вычислению времен релаксации системы и подвижности полярона.

Впервые задача о выравнивании температур компонент в двухкомпонентной системе была рассмотрена в работе [11] (см. изложение подхода Ландау, а также аналогичное рассмотрение релаксации скоростей компонент, например, в книге [12]). В основу исследования в работе [11] было положено кинетическое уравнение для двухкомпонентной плазмы, называемое теперь уравнением Ландау [6]. При вычислении времени релаксации в [11] было сделано предположение, что в компонентах плазмы быстро устанавливается равновесие с некоторыми температурами, которые затем медленно выравниваются. Медленность процесса релаксации связывалась с большим различием масс частиц компонент, что учитывалось в вычислениях как наличие дополнительного малого параметра. При решении описанной задачи для компонент системы без доказательства были использованы функции распределения $(\Phi P)$ Максвелла. Однако в разделе 3 мы показываем, что неравновесная одночастичная ФР газа отличается от распределения Максвелла уже в линейном приближении по малым разностям скоростей и температур поляронного и фононного газов.

Родственная задача в теории полярона рассматривалась также в монографии [2], где в пространственно-однородном случае обсуждалось стационарное решение кинетического уравнения для электрона в слабом электрическом поле и проблема вычисления его подвижности. Следует отметить, что проблема вычисления подвижности является одной из важнейших в теории полярона (см., например, обзор [13]). Раздел 3 завершается сравнением полученных нами результатов с имеющимися в литературе.

\section{2. КИНЕТИЧЕСКОЕ УРАВНЕНИЕ ДЛЯ ПОЛЯРОНА ПРИ НАЛИЧИИ СИЛЬНОГО ЭЛЕКТРИЧЕСКОГО ПОЛЯ}

2.1. Основные уравнения теории. Мы рассматриваем систему $s+b$, состоящую из электрона $s$ (system) и равновесной системы фононов $b$ (bath). Тогда $H_{s+b} \equiv H_{s} \otimes H_{b}$ - пространство состояний системы $s+b$, где $H_{s}, H_{b}$ - пространства состояний систем $s, b$. Статистический оператор $(\mathrm{CO})$ составной системы $\rho(t)$ удовлетворяет квантовому уравнению Лиувилля

$$
\partial_{t} \rho(t)=\mathbf{L} \rho(t), \quad \mathbf{L}=\mathbf{L}_{s}+\mathbf{L}_{b}+\mathbf{L}_{s b}
$$

где операторы Лиувилля $\mathbf{L}, \mathbf{L}_{s}, \mathbf{L}_{b}, \mathbf{L}_{s b}$ определены через операторы $\widehat{H}, \widehat{H}_{s}, \widehat{H}_{b}, \widehat{H}_{s b}$ формулой

$$
\mathbf{L} \rho=-\frac{i}{\hbar}[\widehat{H}, \rho]
$$


$\mathrm{CO} \rho_{s}(t)$ электрона определяется формулой

$$
\operatorname{Sp} \rho(t) \widehat{A}_{s}=\operatorname{Sp}_{s} \rho_{s}(t) \widehat{A}_{s}
$$

где $\widehat{A}_{s}$ - произвольный оператор в $H_{s}, \mathrm{Sp}$ - след в пространстве $H_{s} \otimes H_{b}, \mathrm{Sp}_{s}-$ след в пространстве $H_{s}$. СО $\rho_{s}(t)$ можно записать в виде

$$
\rho_{s}(t)=\widetilde{\operatorname{Sp}}_{b} \rho(t), \quad \operatorname{Sp} \widehat{A}=\operatorname{Sp}_{s} \widetilde{\operatorname{Sp}_{b}} \widehat{A}
$$

где $\widetilde{\mathrm{Sp}}_{b}$ - след по состояниям термостата в пространстве $H_{s} \otimes H_{b}$. Он переводит операторы, действующие в $H_{s} \otimes H_{b}$, в операторы, действующие в $H_{s}$, и отличается от обычного следа в пространстве состояний $H_{b}$, обозначаемого через $\mathrm{Sp}_{b}$.

Будем считать, что по прошествии некоторого времени $\tau_{0}$ система полностью описывается СО электрона $\rho_{s}(t)$ и температурой системы фононов $T_{0}$. В основу рассмотрения положим идею функциональной гипотезы Боголюбова (см., например, книгу [6]):

$$
\rho(t) \underset{t \gg \tau_{0}}{\longrightarrow} \rho\left(\rho_{s}\left(t, \rho_{0}\right)\right) \equiv \rho^{(+)}(t), \quad \rho_{s}(t) \underset{t \gg \tau_{0}}{\longrightarrow} \rho_{s}\left(t, \rho_{0}\right),\left.\quad \rho_{0} \equiv \rho(t)\right|_{t=0} .
$$

Важнейшим утверждением, входящим в функциональную гипотезу, является независимость СО $\rho\left(\rho_{s}\right)$ от начального состояния системы $\rho_{0}$. Мы считаем, что СО $\rho^{(+)}(t)$ удовлетворяют уравнению $(3)$ не только для $t \gg \tau_{0}$, но и для всех $t \geqslant 0$. Это продолжает $\mathrm{CO} \rho_{s}\left(t, \rho_{0}\right)$ на времена $0 \leqslant t \leqslant \tau_{0}$, после чего он удовлетворяет при $t \geqslant 0$ замкнутому кинетическому уравнению

$$
\begin{gathered}
\partial_{t} \rho_{s}\left(t, \rho_{0}\right)=L_{s}\left(\rho_{s}\left(t, \rho_{0}\right)\right) \\
L_{s}\left(\rho_{s}\right) \equiv \mathbf{L}_{s} \rho_{s}+M_{s}\left(\rho_{s}\right), \quad M_{s}\left(\rho_{s}\right) \equiv \widetilde{\operatorname{Sp}_{b}} \mathbf{L}_{s b} \rho\left(\rho_{s}\right)
\end{gathered}
$$

Выпишем уравнения, которые определяют CO $\rho\left(\rho_{s}\right)$. Анализ этих уравнений показывает, что функция $\rho\left(\rho_{s}\right)$ имеет структуру

$$
\rho\left(\rho_{s}\right)=\mathbf{C} w_{b} \rho_{s}
$$

где $\mathbf{C}$ - линейный оператор во множестве операторов, действующих в пространстве $H_{s+b} ; w_{b}-$ равновесный $\mathrm{CO}$ системы фононов ${ }^{1)}$. $\mathrm{CO} \rho\left(\rho_{s}\right)$ согласно предыдущим рассуждениям является решением уравнений

$$
\frac{\partial \rho\left(\rho_{s}\right)}{\partial \rho_{s}} L_{s}\left(\rho_{s}\right)=\mathbf{L} \rho\left(\rho_{s}\right), \quad{\widetilde{\operatorname{Sp}_{b}}} \rho\left(\rho_{s}\right)=\rho_{s} .
$$

Первое из них представляет собой уравнение Лиувилля для СО $\rho\left(\rho_{s}\left(t, \rho_{0}\right)\right)$, в котором производная по времени вычислена с учетом уравнения (8). В (10) производная от оператора по оператору написана для наглядности и может считаться равной

$$
\frac{\partial \rho\left(\rho_{s}\right)}{\partial \rho_{s}}=\mathbf{C} w_{b}
$$

1) По терминологии Пригожина, оператор $\mathbf{C}$, как и оператор Лиувилля $\mathbf{L}$, является супероператором. 
Она является оператором во множестве операторов, действующих в пространстве $H_{s}$ (оператор $L_{s}\left(\rho_{s}\right)$ в (10) относится к тому же классу). Второе уравнение в (10) является следствием определения $(6),(7)$ функции $\rho_{s}\left(t, \rho_{0}\right)$.

Уравнения (10), как известно, имеют не одно решение. Поэтому их следует дополнить некоторым условием, которое называют граничным. Оно должно задавать эволюцию системы $s+b$ в положительном направлении времени. Следуя идеям Боголюбова (см., например, монографию [1]), мы используем условие полного ослабления корреляций, записанное в терминах эволюции системы в положительном направлении времени:

$$
\begin{gathered}
e^{\tau \mathbf{L}_{0}} \rho\left(\rho_{s}\right) \underset{\tau \gg \tau_{0}}{\underset{\tau}{\longrightarrow}} e^{\tau \mathbf{L}_{0}} \rho_{s} w_{b} \\
\mathbf{L}_{0} \equiv \mathbf{L}_{s}+\mathbf{L}_{b}, \quad w_{b}=e^{\left(\Omega-\widehat{H}_{b}\right) / T_{0}}, \quad \operatorname{Sp}_{b} w_{b} \equiv 1
\end{gathered}
$$

где $w_{b}$ - равновесный СО системы фононов $b$. Соотношение (11) выражает принцип пространственного ослабления корреляций, поскольку свободная эволюция разводит электрон и систему фононов и разрывает корреляции между ними. В обоих уравнениях (10), а также в соотношении (11) $\rho_{s}$ является независимой переменной, область определения которой совпадает с областью значений функции $\rho_{s}\left(t, \rho_{0}\right)$.

Первое уравнение в (10) и граничное условие (11) дают следующее интегральное уравнение для $\mathrm{CO} \rho\left(\rho_{s}\right)$ :

$$
\rho\left(\rho_{s}\right)=\rho_{s} w_{b}+\int_{0}^{+\infty} d \tau e^{\tau \mathbf{L}_{0}}\left\{\mathbf{L}_{s b} \rho\left(\rho_{s}\right)-\frac{\partial \rho\left(\rho_{s}\right)}{\partial \rho_{s}} M_{s}\left(\rho_{s}\right)\right\}_{\rho_{s} \rightarrow e^{-\tau \mathbf{L}_{s}} \rho_{s}} .
$$

Это уравнение, а также кинетическое уравнение (8) являются основными результатами развитой нами общей теории. Изложенный в этом разделе подход был применен нами в работе [9] к выводу кинетического уравнения для электрона в полярном кристалле в отсутствие электрического поля.

Заметим, что все результаты настоящего раздела можно сформулировать в терминах оператора С. Если состояние системы описывается СО $\rho$, то электрон согласно (6) находится в состоянии, описываемом $\mathrm{CO} \rho_{s}=\widetilde{\mathrm{Sp}}_{b} \rho$. Это позволяет записать формулу (9) в виде

$$
\rho\left(\rho_{s}\right)=\mathbf{C P} \rho, \quad \mathbf{P} \rho \equiv w_{b}{\widetilde{\mathrm{Sp}_{b}}}_{b},
$$

где введен оператор $\mathbf{P}$, обладающий свойствами

$$
\mathbf{P}^{2}=\mathbf{P}, \quad \mathbf{P L}_{0}=\mathbf{L}_{0} \mathbf{P},
$$

т. е. являющийся проекционным оператором (здесь и ниже в формулах произвольный СО системы $\rho$ справа опускается, и равенства становятся операторными). Соотношения (10) в этих терминах приобретают вид

$$
\text { CPLCP }=\mathbf{L C P}, \quad \mathbf{P C P}=\mathbf{P}
$$

и являются по своему смыслу уравнениями для оператора СP. Единственность их решения обеспечивается граничным условием (11), которое записывается в виде

$$
e^{\tau \mathbf{L}_{0}} \mathbf{C P} \underset{\tau \gg \tau_{0}}{\longrightarrow} e^{\tau \mathbf{L}_{0}} \mathbf{P}
$$


В итоге интегральное уравнение (12) дает интегральное уравнение для оператоpa CP:

$$
\mathbf{C P}=\mathbf{P}+\int_{0}^{+\infty} d \tau e^{\tau \mathbf{L}_{0}}(1-\mathbf{C P}) \mathbf{L}_{s b} \mathbf{C P} e^{-\tau \mathbf{L}_{0}} .
$$

Хотя функция $\rho\left(\rho_{s}\right)$ в обсуждаемой теории линейная, оператор $\mathbf{C P}$ удовлетворяет нелинейному уравнению. Правая часть кинетического уравнения (8) выражается через его решение:

$$
M_{s}\left(\rho_{s}\right)=\widetilde{\operatorname{Sp}_{b}} \mathbf{L}_{s b} \mathbf{C P} w_{b} \rho_{s} .
$$

Функциональная гипотеза (7) в этих терминах приобретает вид

$$
\rho(t) \underset{t \gg \tau_{0}}{\longrightarrow} \rho^{(+)}(t), \quad \rho^{(+)}(t)=\mathbf{C P P} \rho^{(+)}(t),
$$

т. е. СО системы при больших временах определяется своей проекцией. Таким образом, в рассматриваемой задаче метод сокращенного описания допускает проекционную формулировку ${ }^{2}$.

2.2. Кинетическое уравнение для электрона. Перейдем к выводу кинетического уравнения для электрона в теории возмущений по слабому электрон-фононному взаимодействию $\widehat{H}_{s b}$. При этом уравнение $(12)$ легко решается относительно CO $\rho\left(\rho_{s}\right)$ итерационным методом. Одновременно по формуле (8) находится правая часть $L_{s}\left(\rho_{s}\right)$ кинетического уравнения для $\rho_{s}\left(t, \rho_{0}\right)$. Следы по состояниям фононов вычисляются с помощью теоремы Вика-Блоха-де Доминисиса. В итоге после перехода к термодинамическому пределу получим

$$
\begin{gathered}
M_{s}\left(\rho_{s}\right)=\frac{1}{(2 \pi)^{3} \hbar^{2}} \int_{-\infty}^{0} d \tau \int d^{3} k e^{i \omega_{k} \tau}\left\{n_{k}\left[\widehat{A}_{k}^{+}, \rho_{s} \widehat{A}_{k}(\tau)\right]-\right. \\
\left.-\left(1+n_{k}\right)\left[\widehat{A}_{k}^{+}, \widehat{A}_{k}(\tau) \rho_{s}\right]\right\}+ \text { э.c. }+O\left(\lambda^{4}\right),
\end{gathered}
$$

где введены обозначения

$$
n_{k} \equiv \frac{1}{e^{\hbar \omega_{k} / T_{0}}-1}, \quad \widehat{A}_{k}(\tau) \equiv e^{\frac{i}{\hbar} \tau \widehat{H}_{s}} \widehat{A}_{k} e^{-\frac{i}{\hbar} \tau \widehat{H}_{s}}, \quad \lambda \equiv \alpha^{1 / 2}
$$

$\left(n_{k}\right.$ - распределение Планка для подсистемы фононов с температурой $\left.T_{0}\right)$. В полученном выражении (20) для $M_{s}\left(\rho_{s}\right)$ отсутствуют члены порядка $\lambda^{1}, \lambda^{3}$ поскольку они являются средними от нечетного числа операторов $c_{k}, c_{k}^{+}$. Для конкретизации полученных результатов необходимо вычислить оператор $\widehat{A}_{k}(\tau)$. С этой целью заметим, что для оператора координаты электрона в однородном внешнем электрическом поле справедлива формула

$$
\hat{x}_{n}(\tau) \equiv e^{\frac{i}{\hbar} \tau \widehat{H}_{s}} \hat{x}_{n} e^{-\frac{i}{\hbar} \tau \widehat{H}_{s}}=\hat{x}_{n}+\widehat{p}_{n} \frac{\tau}{m}-\frac{e}{m} E_{n} \frac{\tau^{2}}{2},
$$

которая вытекает из выражения $(1)$ для оператора $\widehat{H}_{s}$ и канонических коммутационных соотношений. Оператор $\widehat{A}_{k}(\tau)$ согласно соотношениям $(1)$ и $(21)$ имеет вид

2) Общая проекционная формулировка метода сокращенного описания Боголюбова обсуждается в работе [7]. 
$\widehat{A}_{k}(\tau)=-i g_{k} e^{-i k_{n} \hat{x}_{n}(\tau)}$ и может быть преобразован с помощью тождества Глаубера $e^{\hat{a}+\hat{b}}=e^{\hat{a}} e^{\hat{b}} e^{-\alpha / 2}(\alpha \equiv[\hat{a}, \hat{b}]$ является комплексным числом) к виду

$$
\widehat{A}_{k}(\tau)=-i g_{k} e^{i \frac{k^{2} \hbar \tau}{2 m}} e^{-i k_{n} \hat{x}_{n}} e^{i \frac{e \tau^{2}}{2 m} k_{l} E_{l}} e^{-i \frac{\tau}{m} k_{s} \hat{p}_{s}} .
$$

В координатном представлении этот оператор дается формулой

$$
\left\langle\vec{x}\left|\widehat{A}_{k}(\tau)\right| \vec{x}^{\prime}\right\rangle=-i g_{k} e^{i \frac{k^{2} \hbar \tau}{2 m}} e^{-i k_{n} x_{n}} e^{i \frac{e \tau^{2}}{2 m} k_{l} E_{l}} \delta\left(\vec{x}-\vec{x}^{\prime}-\frac{k \hbar}{m} \tau\right),
$$

здесь $\hat{x}_{n}|\vec{x}\rangle \equiv x_{n}|\vec{x}\rangle,\left\langle\vec{x} \mid \vec{x}^{\prime}\right\rangle \equiv \delta\left(\vec{x}-\vec{x}^{\prime}\right)$. Соотношения (8), (20) дают выведенное нами кинетическое уравнение как уравнение для статистического оператора электрона $\rho_{s}\left(t, \rho_{0}\right)$. Перепишем это уравнение в более привычной форме - в терминах вигнеровской ФР электрона, определяемой как

$$
f_{p}(\vec{x}, t)=\int d^{3} x^{\prime}\left\langle\vec{x}-\frac{\vec{x}^{\prime}}{2}\left|\rho_{s}\left(t, \rho_{0}\right)\right| \vec{x}+\frac{\vec{x}^{\prime}}{2}\right\rangle e^{\frac{i}{\hbar} \vec{p} \vec{x}^{\prime}}
$$

Простые преобразования приводят к кинетическому уравнению

$$
\partial_{t} f_{p}(\vec{x}, t)=-\frac{p_{n}}{m} \frac{\partial f_{p}(\vec{x}, t)}{\partial x_{n}}+e E_{n} \frac{\partial f_{p}(\vec{x}, t)}{\partial p_{n}}+I_{p}(\vec{x}, f(t)),
$$

где интеграл столкновений $I_{p}(\vec{x}, f)$ дается формулой

$$
I_{p}(\vec{x}, f) \equiv \int d^{3} x^{\prime}\left\langle\vec{x}-\frac{\vec{x}^{\prime}}{2}\left|M\left(\rho_{s}\right)\right| \vec{x}+\frac{\vec{x}^{\prime}}{2}\right\rangle e^{\frac{i}{\hbar} \vec{p}^{\prime}} .
$$

Вычисление интеграла столкновений с точностью, определяемой выражением (20), т. е. в основном порядке теории возмущений дает

$$
\begin{aligned}
& I_{p}(\vec{x}, f)=\frac{1}{4 \pi^{3} \hbar^{2}} \int_{-\infty}^{0} d \tau \int d^{3} k g_{k}^{2}\left\{n_{k} f_{p-k \hbar}\left(\vec{x}+\vec{k} \frac{\hbar \tau}{2 m}\right)-\left(1+n_{k}\right) f_{p}\left(\vec{x}-\vec{k} \frac{\hbar \tau}{2 m}\right)\right\} \times \\
& \quad \times \cos \frac{\tau}{\hbar}\left(\varepsilon_{p-k \hbar}+\hbar \omega_{k}-\varepsilon_{p}+\vec{k} \vec{E} e \frac{\hbar \tau}{2 m}\right)+ \\
& \quad+\frac{1}{4 \pi^{3} \hbar^{2}} \int_{-\infty}^{0} d \tau \int d^{3} k g_{k}^{2}\left\{\left(1+n_{k}\right) f_{p+k \hbar}\left(\vec{x}-\vec{k} \frac{\hbar \tau}{2 m}\right)-n_{k} f_{p}\left(\vec{x}+\vec{k} \frac{\hbar \tau}{2 m}\right)\right\} \times \\
& \quad \times \cos \frac{\tau}{\hbar}\left(\varepsilon_{p}+\hbar \omega_{k}-\varepsilon_{p+k \hbar}+\vec{k} \vec{E} e \frac{\hbar \tau}{2 m}\right)+O\left(\lambda^{4}\right) .
\end{aligned}
$$

Кинетическое уравнение (26) с интегралом столкновений (28) (наряду с общей теорией, развитой в предыдущем пункте) является основным результатом данного раздела $^{3)}$ и обобщает кинетическое уравнение, полученное в нашей работе [9], на случай наличия сильного электрического поля. Оно описывает электрон в кристалле в приближении слабого взаимодействия, но без предположения о слабой неоднородности его состояния. Поправка к полученному интегралу столкновений имеет

3) Вывод кинетического уравнения с интегралом столкновений (28) был доложен на конференции [14]. 
четвертый порядок малости вследствие того, что вклад третьего порядка является средним от нечетного числа бозевских операторов фононов и согласно теореме Вика-Блоха-де Доминисиса равен нулю. Отметим также, что в этом разделе соотношения (2) не используются.

В слабо неоднородном состоянии электрона его вигнеровская $\Phi \mathrm{P} f_{p}(\vec{x})$ мало меняется с изменением $\vec{x}$, и в $(28)$ можно пренебречь добавками к $\vec{x}$. При этом интеграл столкновений приобретает вид

$$
\begin{aligned}
I_{p}(\vec{x}, f)= & \frac{1}{(2 \pi)^{2} \hbar} \int d^{3} k g_{k}^{2}\left\{n_{k} f_{p-k \hbar}(\vec{x})-\left(1+n_{k}\right) f_{p}(\vec{x})\right\} \times \\
& \times \delta\left(\varepsilon_{p-k \hbar}+\hbar \omega_{k}-\varepsilon_{p} ; \frac{e \hbar^{2}}{2 m} \vec{k} \vec{E}\right)+ \\
& +\frac{1}{(2 \pi)^{2} \hbar} \int d^{3} k g_{k}^{2}\left\{\left(1+n_{k}\right) f_{p+k \hbar}(\vec{x})-n_{k} f_{p}(\vec{x})\right\} \times \\
& \times \delta\left(\varepsilon_{p}+\hbar \omega_{k}-\varepsilon_{p+k \hbar} ; \frac{e \hbar^{2}}{2 m} \vec{k} \vec{E}\right)+O\left(\lambda^{4}\right),
\end{aligned}
$$

где стоят "размытые" $\delta$-функции, определяемые формулой

$$
\begin{aligned}
\delta(x ; \alpha)=\frac{1}{\pi} \int_{-\infty}^{0} d \tau \cos \left(x \tau+\alpha \tau^{2}\right) & =\frac{1}{2 \sqrt{2 \pi|x|}}\left(\cos \frac{\alpha^{2}}{4|x|}+\sin \frac{\alpha^{2}}{4|x|}\right), \\
\lim _{\alpha \rightarrow 0} \delta(x ; \alpha) & =\delta(x)
\end{aligned}
$$

(значение интеграла приведено в справочнике [15]). При отсутствии электрического поля интеграл имеет больцмановский вид и обычным образом учитывает влияние процессов излучения и поглощения фононов электроном на число электронов с импульсом $p$. В этих процессах сохраняется энергия, что описывается обычными $\delta$-функциями.

\section{3. РЕЛАКСАЦИЯ СКОРОСТИ И ТЕМПЕРАТУРЫ ПОЛЯРОННОГО ГАЗА МАЛОЙ ПЛОТНОСТИ}

3.1. Обобщение метода Чепмена-Энскога. Кинетическое уравнение для одного полярона (26), (28) совпадает с кинетическим уравнением для поляронного газа малой плотности, поскольку в этом пределе не проявляются фермиевские свойства электронов. Поэтому в настоящем разделе для рассмотрения пространственно-однородного поляронного газа при наличии слабого электрического поля мы исходим согласно (26), (29) из кинетического уравнения

$$
\partial_{t} f_{p}(t)=\mathcal{L} f_{p}(t), \quad \mathcal{L} \equiv \mathcal{L}_{0}+e E_{l} \frac{\partial}{\partial p_{l}}, \quad \mathcal{L}_{0} f_{p} \equiv \int d^{3} p^{\prime} M\left(p, p^{\prime}\right) f_{p^{\prime}},
$$

где

$$
\begin{aligned}
& M\left(p, p^{\prime}\right) \equiv \frac{1}{(2 \pi)^{2} \hbar} \int d^{3} k g_{k}^{2}\left\{n_{k} \delta\left(p^{\prime}-[p-k \hbar]\right)-\left(1+n_{k}\right) \delta\left(p^{\prime}-p\right)\right\} \times \\
& \quad \times \delta\left(\varepsilon_{p-k \hbar}+\hbar \omega_{k}-\varepsilon_{p}\right)+\frac{1}{(2 \pi)^{2} \hbar} \int d^{3} p g_{k}^{2}\left\{\left(1+n_{k}\right) \delta\left(p^{\prime}-[p+k \hbar]\right)-n_{k} \delta\left(p^{\prime}-p\right)\right\} \times \\
& \quad \times \delta\left(\varepsilon_{p}+\hbar \omega_{k}-\varepsilon_{p+k \hbar}\right) .
\end{aligned}
$$


Это кинетическое уравнение получено Боголюбовым методом исключения бозонных переменных (см. ссылки в книге [2]) и методом сокращенного описания в нашей работе [9].

Рассмотрим такие неравновесные состояния поляронного газа в кристалле, которые полностью описываются его скоростью $u_{l}(t)$ и температурой $T(t)$. Они наступают по прошествии некоторого времени $\tau_{1}$, которое зависит от начального состояния системы, описываемого $\Phi \mathrm{P} f_{p}(0) \equiv f_{p, 0}$. Задачу построения описания состояния электрона с помощью этих параметров мы решаем на основе метода сокращенного описания Боголюбова, исходя из его идеи функциональной гипотезы

$$
f_{p}(t) \underset{t \gg \tau_{1}}{\longrightarrow} f_{p}(T(t), u(t)) .
$$

При этом температура $T(t)$ и скорость $u(t)$ газа поляронов определяются формулами

$$
\int d^{3} p f_{p}(t) p_{l} \underset{t \gg \tau_{1}}{\longrightarrow} m n u_{l}(t), \quad \int d^{3} p f_{p}(t) \varepsilon_{p} \underset{t \gg \tau_{1}}{\longrightarrow} \frac{3}{2} n T(t)+\frac{1}{2} m n u(t)^{2},
$$

в которых $n-$ постоянная плотность поляронов,

$$
\int d^{3} p f_{p}(t)=n
$$

В соотношении (33) функция $f_{p}(T, u)$ не зависит от начального состояния системы, описываемого $\Phi \mathrm{P} f_{p, 0}$. Фактически такой подход ведет к некоторому обобщению метода Чепмена-Энскога. В своей стандартной формулировке (см., например, [6], [10]) этот метод ориентирован на исследование гидродинамической эволюции. Обобщения метода Чепмена-Энскога возможны на основе его понимания как частного случая метода сокращенного описания, примененного к решению кинетического уравнения. Задача настоящего раздела состоит в описании процессов релаксации в системе, в которых $u_{l}(t) \underset{t \gg \tau_{u}}{\longrightarrow} 0, T(t) \underset{t \gg \tau_{T}}{\longrightarrow} T_{0}$, где $\tau_{u}, \tau_{T}-$ времена релаксации. При этом предполагается, что скорость фононного газа равна нулю, а $T_{0}$ является его температурой.

В соответствии с методом сокращенного описания неравновесных состояний функция $f_{p}(T(t), u(t))$ является точным решением кинетического уравнения $(31)$ :

$$
\partial_{t} f_{p}(T(t), u(t))=\mathcal{L} f_{p}(T(t), u(t)), \quad t \gg \tau_{0} .
$$

Функциональная гипотеза (33) приводит к замкнутым уравнениям для величин $T(t)$, $u_{l}(t)$ :

$$
\partial_{t} T(t)=L_{0}(T(t), u(t)), \quad \partial_{t} u_{l}(t)=L_{l}(T(t), u(t)), \quad t \gg \tau_{0},
$$

где

$$
\begin{aligned}
L_{0}(T, u) & =\frac{2}{3 n} \int d^{3} p\left(\varepsilon_{p}-p_{l} u_{l}\right) \mathcal{L}_{0} f_{p}(T, u), \\
L_{l}(T, u) & =-\frac{e}{m} E_{l}+\frac{1}{m n} \int d^{3} p p_{l} \mathcal{L}_{0} f_{p}(T, u) .
\end{aligned}
$$

Согласно $(36),(37) \Phi \mathrm{P} f_{p}(T, u)$ удовлетворяет следующему кинетическому уравнению при сокращенном описании:

$$
\frac{\partial f_{p}(T, u)}{\partial T} L_{0}(T, u)+\frac{\partial f_{p}(T, u)}{\partial u_{l}} L_{l}(T, u)=\mathcal{L} f_{p}(T, u) .
$$


Определение параметров сокращенного описания (34) и функциональная гипотеза (33) ведут к дополнительным условиям на функцию $f_{p}(T, u)$ :

$$
\int d^{3} p f_{p}(T, u) p_{l}=m n u_{l}, \quad \int d^{3} p f_{p}(T, u) \varepsilon_{p}=\frac{3}{2} n T+\frac{m u^{2}}{2} .
$$

Полученные уравнения (39), (40) являются основными уравнениями метода сокращенного описания для вычисления $\Phi \mathrm{P} f_{p}(T, u)$.

Изучим явления в системе вблизи равновесия, считая разность температур $T-T_{0}$ поляронного газа и фононной подсистемы, скорость поляронного газа $u_{l}$ и электрическое поле $E_{l}$ малыми величинами одного порядка $\varepsilon$. Мы ищем $\Phi \mathrm{P} f_{p}(T, u)$ в виде разложения по $\varepsilon$ :

$$
f_{p}(T, u)=f_{p}^{(0)}+f_{p}^{(1)}+O\left(\varepsilon^{2}\right) .
$$

Анализ уравнений $(39),(40)$ с учетом соображений вращательной инвариантности и определения $(31)$ оператора $\mathcal{L}_{0}$ показывает, что

$$
\begin{aligned}
& f_{p}^{(0)}=w_{p}\left(\beta_{0}\right), \quad w_{p}(\beta) \equiv n\left(\frac{\beta}{2 \pi m}\right)^{3 / 2} e^{-\beta \varepsilon_{p}}, \\
& f_{p}^{(1)}=w_{p}\left(\beta_{0}\right)\left\{A\left(\beta_{0} \varepsilon_{p}\right) p_{l} u_{l}+B\left(\beta_{0} \varepsilon_{p}\right)\left(T-T_{0}\right)+C\left(\beta_{0} \varepsilon_{p}\right) p_{l} E_{l}\right\},
\end{aligned}
$$

где $A(x), B(x), C(x)$ - некоторые скалярные функции; $w_{p}(\beta)$ - распределение Максвелла $\left(\mathcal{L}_{0} w_{p}\left(\beta_{0}\right)=0\right) ; \beta_{0} \equiv T_{0}^{-1}, \beta \equiv T^{-1}$. Интегральные уравнения для $A(x), B(x)$, $C(x)$ удобно записать, вводя вместо ядра $M\left(p, p^{\prime}\right)$ оператора $\mathcal{L}_{0}$ функцию $K\left(p, p^{\prime}\right)$, определяемую формулой

$$
M\left(p, p^{\prime}\right) w_{p^{\prime}}\left(\beta_{0}\right)=-w_{p}\left(\beta_{0}\right) K\left(p, p^{\prime}\right) .
$$

В этих терминах функции $A(x), B(x), C(x)$ являются решениями интегральных уравнений

$$
\begin{aligned}
\int d^{3} p^{\prime} K\left(p, p^{\prime}\right) A\left(\beta_{0} \varepsilon_{p^{\prime}}\right) p_{l}^{\prime} & =\lambda_{u} A\left(\beta_{0} \varepsilon_{p}\right) p_{l}, \\
\int d^{3} p^{\prime} K\left(p, p^{\prime}\right) B\left(\beta_{0} \varepsilon_{p^{\prime}}\right) & =\lambda_{T} B\left(\beta_{0} \varepsilon_{p}\right), \\
\int d^{3} p^{\prime} K\left(p, p^{\prime}\right) C\left(\beta_{0} \varepsilon_{p^{\prime}}\right) p_{l}^{\prime} & =\left\{\gamma A\left(\beta_{0} \varepsilon_{p}\right)-\frac{e \beta_{0}}{m}\right\} p_{l}
\end{aligned}
$$

с дополнительными условиями

$$
\begin{aligned}
& \int d^{3} p w_{p}\left(\beta_{0}\right) \varepsilon_{p} A\left(\beta_{0} \varepsilon_{p}\right)=\frac{3}{2}, \quad \int d^{3} p w_{p}\left(\beta_{0}\right) \varepsilon_{p} B\left(\beta_{0} \varepsilon_{p}\right)=\frac{3}{2}, \\
& \int d^{3} p w_{p}\left(\beta_{0}\right) B\left(\beta_{0} \varepsilon_{p}\right)=0, \quad \int d^{3} p w_{p}\left(\beta_{0}\right) \varepsilon_{p} C\left(\beta_{0} \varepsilon_{p}\right)=0 .
\end{aligned}
$$

В уравнения (45) входят коэффициенты $\lambda_{u}, \lambda_{T}, \gamma$, которые будут найдены при их решении. Эти коэффициенты определяют временнь́е уравнения для скорости $u_{n}$ и температуры $T$ поляронного газа:

$$
\partial_{t} u_{l}=-\lambda_{u} u_{l}-\gamma E_{l}+O\left(\varepsilon^{2}\right), \quad \partial_{t} T=-\lambda_{T}\left(T-T_{0}\right)+O\left(\varepsilon^{2}\right) .
$$


При этом $\tau_{T} \equiv \lambda_{T}^{-1}$ и $\tau_{u} \equiv \lambda_{u}^{-1}$ являются временами релаксации температуры и скорости, а коэффициент $\mu=\gamma / \lambda_{u}$ определяет подвижность полярона в стационарном состоянии. Для них согласно (38), (43) справедливы выражения

$$
\begin{gathered}
\lambda_{u}=\frac{1}{3 m}\left\{p_{l}, A\left(\beta_{0} \varepsilon_{p}\right) p_{l}\right\}, \quad \lambda_{T}=\frac{2}{3}\left\{\varepsilon_{p}, B\left(\beta_{0} \varepsilon_{p}\right)\right\}, \\
\gamma=\frac{1}{3 m}\left\{p_{l}, C\left(\beta_{0} \varepsilon_{p}\right) p_{l}\right\}+\frac{e}{m},
\end{gathered}
$$

где введена билинейная форма

$$
\begin{aligned}
\left\{A_{p}, B_{p}\right\} \equiv & \int d^{3} p \int d^{3} p^{\prime} w_{p}\left(\beta_{0}\right) A_{p} K\left(p, p^{\prime}\right) B_{p^{\prime}}= \\
= & \frac{1}{(2 \pi)^{2} \hbar} \int d^{3} p \int d^{3} k g_{k}^{2} n_{k} w_{p}\left(\beta_{0}\right)\left\{A_{p}-A_{p+k \hbar}\right\}\left\{B_{p}-B_{p+k \hbar}\right\} \times \\
& \quad \times \delta\left(\varepsilon_{p}-\varepsilon_{p+k \hbar}+\omega_{k}\right),
\end{aligned}
$$

аналоги которой широко используются в кинетической теории (см., например, [10]), поскольку она обладает полезными свойствами:

$$
\left\{A_{p}, B_{p}\right\}=\left\{B_{p}, A_{p}\right\}, \quad\left\{A_{p}, A_{p}\right\} \geqslant 0, \quad\left\{A_{p}, A_{p}\right\}=0 \Rightarrow A_{p}=\text { const }
$$

где $A_{p}, B_{p}$ - произвольные функции. Отметим, что соотношения (48) не делают уравнения (45) нелинейными. Эти формулы являются следствием интегральных уравнений (45) и дополнительных условий (46). Функции $A\left(\beta_{0} \varepsilon_{p}\right) p_{l}, B\left(\beta_{0} \varepsilon_{p}\right)$ суть собственные функции интегрального оператора с ядром $K\left(p, p^{\prime}\right)$, отвечающими собственным значениям $\lambda_{u}, \lambda_{T}$. В этом смысле собственные значения являются функциями от собственных функций.

Заметим, что изложенное обобщение метода Чепмена-Энскога позволяет в следующих порядках теории возмущений по $\varepsilon$ выйти за рамки линейной теории и получить нелинейные уравнения для $T(t), u_{l}(t)$.

3.2. Приближенное решение интегральных уравнений теории. Перейдем к приближенному решению уравнений (45), (46). Будем искать их решение в виде ряда по полиномам Сонина $S_{\alpha}^{n}(x)$ (см., например, книгу [10]) при $\alpha=1 / 2,3 / 2$ :

$$
A(x)=\sum_{n=0}^{\infty} a_{n} S_{3 / 2}^{n}(x), \quad B(x)=\sum_{n=0}^{\infty} b_{n} S_{1 / 2}^{n}(x), \quad C(x)=\sum_{n=0}^{\infty} c_{n} S_{3 / 2}^{n}(x) .
$$

Выбор именно этих ортогональных полиномов мотивирован структурой уравнений (45), (46), соображениями вращательной инвариантности и условиями ортонормировки полиномов Сонина, которые можно записать в виде

$$
\begin{aligned}
\int d^{3} p w_{p}\left(\beta_{0}\right) p^{2} S_{3 / 2}^{n}\left(\beta_{0} \varepsilon_{p}\right) S_{3 / 2}^{n^{\prime}}\left(\beta_{0} \varepsilon_{p}\right) & =x_{n} \delta_{n^{\prime} n}, & x_{n} & \equiv \frac{4 m}{\beta_{0} \sqrt{\pi}} \frac{\Gamma(n+5 / 2)}{n !} ; \\
\int d^{3} p w_{p}\left(\beta_{0}\right) S_{1 / 2}^{n}\left(\beta_{0} \varepsilon_{p}\right) S_{1 / 2}^{n^{\prime}}\left(\beta_{0} \varepsilon_{p}\right) & =y_{n} \delta_{n^{\prime} n}, & y_{n} & \equiv \frac{2}{\sqrt{\pi}} \frac{\Gamma(n+3 / 2)}{n !} .
\end{aligned}
$$


Тогда уравнения (45) и дополнительные условия (46) дают

$$
\begin{aligned}
\sum_{n^{\prime}=0}^{\infty} A_{n n^{\prime}} a_{n^{\prime}} \sqrt{x_{n^{\prime}}}=\lambda_{u} a_{n} \sqrt{x_{n}}, & a_{0}=\beta_{0} ; \\
\sum_{n^{\prime}=0}^{\infty} B_{n n^{\prime}} b_{n^{\prime}} \sqrt{y_{n^{\prime}}}=\lambda_{T} b_{n} \sqrt{y_{n}}, & b_{0}=0, \quad b_{1}=-\beta_{0} ; \\
\sum_{n^{\prime}=0}^{\infty} A_{n n^{\prime}} c_{n^{\prime}} \sqrt{x_{n^{\prime}}}=\gamma a_{n} \sqrt{x_{n}}-e\left(\frac{3 \beta_{0}}{m}\right)^{1 / 2} \delta_{n, 0}, & c_{0}=0,
\end{aligned}
$$

где введены симметричные положительно определенные матрицы

$$
\begin{aligned}
& A_{n n^{\prime}}=\left\{p_{l} S_{3 / 2}^{n}\left(\beta_{0} \varepsilon_{p}\right), p_{l} S_{3 / 2}^{n^{\prime}}\left(\beta_{0} \varepsilon_{p}\right)\right\}\left(x_{n} x_{n^{\prime}}\right)^{-1 / 2}, \\
& B_{n n^{\prime}}=\left\{S_{1 / 2}^{n}\left(\beta_{0} \varepsilon_{p}\right), S_{1 / 2}^{n^{\prime}}\left(\beta_{0} \varepsilon_{p}\right)\right\}\left(y_{n} y_{n^{\prime}}\right)^{-1 / 2}
\end{aligned}
$$

(согласно (49), (55) $\left.B_{0 n}=B_{n 0}=0\right)$.

Первые два уравнения в (53) являются уравнениями на собственные векторы $\tilde{a}_{n} \equiv a_{n} \sqrt{x_{n}}, \tilde{b}_{n} \equiv b_{n} \sqrt{y_{n}}$ и собственные значения $\lambda_{u}, \lambda_{T}$ матриц $A_{n n^{\prime}}, B_{n n^{\prime}}$. Они определяют кинетические моды системы. Собственные значения $\lambda_{u}, \lambda_{T}$ формально являются решениями уравнений

$$
\operatorname{det}\left\|A_{n n^{\prime}}-\lambda_{u} \delta_{n n^{\prime}}\right\|=0, \quad \operatorname{det}\left\|B_{n n^{\prime}}-\lambda_{T} \delta_{n n^{\prime}}\right\|=0 .
$$

Эти уравнения имеют бесконечное множество решений, среди которых следует выбрать минимальные. Именно они описывает самый медленный процесс релаксации. При этом дополнительные условия в (53) определяют собственные векторы однозначно. Третье соотношение в (53) является системой линейных уравнений относительно величин $c_{n}(n \geqslant 1)$ и $\gamma$.

Ограничимся при решении уравнений традиционными приближениями одногодвух полиномов, учитывая, что

$$
S_{\alpha}^{0}(x)=1, \quad S_{\alpha}^{1}(x)=-x+1+\alpha, \quad S_{\alpha}^{2}(x)=\frac{x^{2}-2(2+\alpha) x+(2+\alpha)(1+\alpha)}{2} .
$$

В результате получим следующие выражения для функций $A(x), B(x), C(x)$ и коэффициентов $\lambda_{u}, \lambda_{T}, \gamma$. Приближение одного полинома для $A(x)$ :

$$
A(x)=a_{0}, \quad a_{0}=\beta_{0}, \quad \lambda_{u}=A_{00} .
$$

Приближение двух полиномов для $A(x)$ :

$$
\begin{gathered}
A(x)=a_{0}+a_{1} S_{3 / 2}^{1}(x), \\
a_{0}=\beta_{0}, \quad a_{1}=\frac{\lambda_{u}-A_{00}}{A_{11}} \beta_{0}\left(\frac{2}{5}\right)^{1 / 2}, \\
\lambda_{u}=\frac{A_{00}+A_{11}-\left[\left(A_{00}-A_{11}\right)^{2}+4 A_{01}^{2}\right]^{1 / 2}}{2} .
\end{gathered}
$$

Приближение одного полинома для $B(x)$ :

$$
B(x)=b_{1} S_{1 / 2}^{1}\left(\beta_{0} \varepsilon_{p}\right), \quad b_{1}=-\beta_{0}, \quad \lambda_{T}=B_{11} .
$$


Приближение двух полиномов для $B(x)$ :

$$
\begin{gathered}
B(x)=b_{1} S_{1 / 2}^{1}(x)+b_{2} S_{1 / 2}^{2}(x), \\
b_{1}=-\beta_{0}, \quad b_{2}=\frac{B_{11}-\lambda_{T}}{B_{12}} \beta_{0}\left(\frac{4}{5}\right)^{1 / 2}, \\
\lambda_{T}=\frac{B_{11}+B_{22}-\left[\left(B_{11}-B_{22}\right)^{2}+4 B_{12}^{2}\right]^{1 / 2}}{2} .
\end{gathered}
$$

Приближение одного полинома для $C(x)$ :

$$
C(x)=c_{1} S_{3 / 2}^{1}(x), \quad c_{1}=\gamma \frac{a_{1}}{A_{11}}, \quad \gamma=\frac{e}{m} \frac{\beta_{0} A_{11}}{\beta_{0} A_{11}-(5 / 2)^{1 / 2} a_{1} A_{01}} .
$$

Приближение двух полиномов для $C(x)$ :

$$
\begin{gathered}
C(x)=c_{1} S_{3 / 2}^{1}(x)+c_{2} S_{3 / 2}^{2}(x), \\
c_{1}=\gamma \frac{a_{1} A_{22}}{A_{11} A_{22}-A_{12}^{2}}, \quad c_{2}=-\gamma \frac{a_{1} A_{12}}{A_{11} A_{22}-A_{12}^{2}}, \\
\gamma=\frac{e}{m} \frac{\beta_{0}\left(A_{11} A_{22}-A_{12}^{2}\right)}{\beta_{0}\left(A_{11} A_{22}-A_{12}^{2}\right)-(5 / 2)^{1 / 2} a_{1}\left(A_{01} A_{22}-A_{02} A_{21}\right)} .
\end{gathered}
$$

Из полученных результатов вытекает, что неравновесная $\Phi Р$ системы $f_{p}(T, u)$ уже в линейном приближении по малой скорости $u_{n}$ и малой разности температур $T-T_{0}$ отличается от распределения Максвелла $w_{p-m и}(\beta)$. А именно, согласно соотношениям (59)-(61) справедлива формула

$$
f_{p}(T, u) \neq w_{p-m u}(\beta)+O\left(\varepsilon^{2}\right) .
$$

Равенство в ней имеет место только в приближении одного полинома для $a_{n}$ и $b_{n}$, когда

$$
\begin{aligned}
& A(x)=\beta_{0}, \quad B(x)=\beta_{0}\left(x-\frac{3}{2}\right), \quad C(x)=0 \\
& \lambda_{u}=\frac{\beta_{0}}{3 m}\left\{p_{l}, p_{l}\right\}, \quad \lambda_{T}=\frac{2 \beta_{0}^{2}}{3}\left\{\varepsilon_{p}, \varepsilon_{p}\right\}, \quad \gamma=\frac{e}{m} .
\end{aligned}
$$

Значение коэффициента $\gamma$ в (63) соответствует свободному электрону во внешнем электрическом поле, что ясно из второго закона Ньютона (см. уравнения (47)). Приближение одного полинома (63) может быть названо приближением Ландау, поскольку при анализе релаксации температур в двухкомпонентной плазме в известной работе [11] было использовано в качестве неравновесной $\Phi \mathrm{P} f_{p}(T, u)$ распределение Максвелла с $T \neq T_{0}$ (релаксация скоростей рассмотрена, например, в книге [12]). В настоящей работе мы изучаем двухкомпонентную систему в рамках иного подхода, основанного на методе сокращенного описания Боголюбова.

Конечно, вопросы сходимости изложенной в этом разделе приближенной процедуры вычисления функций $A(x), B(x), C(x)$ и коэффициентов $\lambda_{u}, \lambda_{T}, \gamma$ требуют своего исследования. Заметим в этой связи, что анализ интегрального уравнения (45) для $C(x)$ упрощается, если использовать вариационный принцип Колера, вытекающий из соотношений (50) (см., например, монографию [16]). В то же время исследование и этого уравнения сводится к изучению спектральной задачи для интегрального оператора с ядром $K\left(p, p^{\prime}\right)$.

6 Теоретическая и математическая физика, т. 168, № 2, 2011 г. 
3.3. Подвижность полярона: обсуждение результатов. Согласно соотношениям (50) в стационарном состоянии системы $T=T_{0}, u_{l}=-\mu E_{l}$, где $\mu \equiv \gamma / \lambda_{u}-$ подвижность полярона. При этом ток в системе имеет вид $j_{l}=\sigma E_{l}$, где $\sigma \equiv e n \mu-$ проводимость. Полученные в предыдущем разделе результаты будут проанализированы в полной мере в другом месте. Здесь же мы ограничимся сопоставлением приближения одного полинома и результатов некоторых других подходов к вычислению подвижности полярона в модели Фрёлиха.

В нашей статье [17] показано, что коэффициенты релаксации $\lambda_{u}, \lambda_{T}$ в приближении (63) и при учете формул (2) выражаются через функции Макдональда $K_{\alpha}(x)$ :

$$
\lambda_{u}=\frac{2^{3 / 2} \alpha \omega_{0}}{3 \pi^{1 / 2}} \frac{\nu^{3 / 2}}{\operatorname{sh}(\nu / 2)} K_{1}\left(\frac{\nu}{2}\right), \quad \lambda_{T}=\frac{2^{3 / 2} \alpha \omega_{0}}{3 \pi^{1 / 2}} \frac{\nu^{5 / 2}}{\operatorname{sh}(\nu / 2)} K_{0}\left(\frac{\nu}{2}\right), \quad \nu \equiv \frac{\hbar \omega_{0}}{T_{0}},
$$

где учтено равенство [15]

$$
\int_{0}^{\infty} d x x^{\alpha-1} e^{-p x} e^{-q / x}=2 K_{\alpha}(2 \sqrt{p q})\left(\frac{q}{p}\right)^{\alpha / 2} .
$$

В случае низких температур $\nu \gg 1$ эти выражения можно упростить с учетом разложений функций $K_{\alpha}(x)$ при $x \rightarrow+\infty[15]:$

$$
K_{\alpha}(x)=\sqrt{\frac{\pi}{2 x}} e^{-x}\left[1+O\left(\frac{1}{x}\right)\right], \quad \alpha=0,1 .
$$

В результате при низких температурах мы получаем следующие формулы:

$$
\begin{array}{ll}
\lambda_{u} & =\frac{2^{5 / 2} \alpha \omega_{0}}{3} \nu^{1 / 2} e^{-\nu}\left[1+O\left(\frac{1}{\nu}\right)\right], \\
\lambda_{T} & =\frac{2^{5 / 2} \alpha \omega_{0}}{3} \nu^{3 / 2} e^{-\nu}\left[1+O\left(\frac{1}{\nu}\right)\right],
\end{array}
$$

В случае высоких температур $\nu \ll 1$ эти выражения можно упростить с учетом разложений функций $K_{\alpha}(x)$ при $x \rightarrow 0$ [15],

$$
K_{0}(x)=\ln x+O(1), \quad K_{1}(x)=\frac{1}{x}+O(x \ln x) .
$$

В итоге коэффициенты релаксации при высоких температурах даются следующими формулами [17]:

$$
\begin{aligned}
& \lambda_{u}=\frac{2^{7 / 2} \alpha \omega_{0}}{3 \pi^{1 / 2}} \nu^{-1 / 2}[1+O(\nu)], \\
& \lambda_{T}=\frac{2^{5 / 2} \alpha \omega_{0}}{3 \pi^{1 / 2}} \nu^{3 / 2}[\ln \nu+O(1)],
\end{aligned}
$$

Формулы (67), (69) показывают, что в обоих предельных случаях температуры подсистем выравниваются медленно. Это же справедливо для скорости поляронного газа при низких температурах, однако при высоких температурах скорость затухает быстро. В указанных предельных случаях подвижность полярона имеет вид

$$
\begin{aligned}
& \mu=\frac{3 e}{2^{5 / 2} m \alpha \omega_{0}} \nu^{-1 / 2} e^{\nu}\left[1+O\left(\frac{1}{\nu}\right)\right], \quad \nu \gg 1, \\
& \mu=\frac{3 \pi^{1 / 2} e}{2^{7 / 2} m \alpha \omega_{0}} \nu^{1 / 2}[1+O(\nu)], \quad \nu \ll 1 .
\end{aligned}
$$


Классический подход к вычислению подвижности принадлежит Лоренцу и является вариантом метода Чепмена-Энскога для стационарных состояний. В наших обозначениях (43), (45) такой подход дает

$$
f_{p}^{(1)}=w_{p}\left(\beta_{0}\right) C\left(\beta_{0} \varepsilon_{p}\right) p_{l} E_{l}, \quad \int d^{3} p^{\prime} K\left(p, p^{\prime}\right) C\left(\beta_{0} \varepsilon_{p^{\prime}}\right) p_{l}^{\prime}=-\frac{e \beta_{0}}{m} p_{l} .
$$

Это интегральное уравнение для $C(x)$ решается в приближении времени релаксации

$$
\int d^{3} p^{\prime} K\left(p, p^{\prime}\right) C\left(\beta_{0} \varepsilon_{p^{\prime}}\right) p_{l}^{\prime} \approx C\left(\beta_{0} \varepsilon_{p}\right) \frac{p_{l}}{\tau\left(\beta_{0} \varepsilon_{p}\right)},
$$

где время релаксации $\tau\left(\beta_{0} \varepsilon_{p}\right)$ определяется формулой

$$
\int d^{3} p^{\prime} K\left(p, p^{\prime}\right) p_{l}^{\prime} \equiv \frac{p_{l}}{\tau\left(\beta_{0} \varepsilon_{p}\right)} .
$$

При этом подвижность электрона дается выражением

$$
\mu=\frac{e \beta_{0}}{3 m^{2}} \int d^{3} p w_{p}\left(\beta_{0}\right) p^{2} \tau\left(\beta_{0} \varepsilon_{p}\right),
$$

для использования которого нужно вычислить время релаксации $\tau\left(\beta_{0} \varepsilon_{p}\right)$. Это приближение применимо, если энергия полярона мало изменяется в процессах, описываемых ядром $K\left(p, p^{\prime}\right)$. В случае неупругих процессов используется вариационный принцип Колера ${ }^{4)}$. Является общепринятым (см., например, сборник [13]), что приближение времени релаксации справедливо в модели Фрёлиха при высоких температурах. В книге [18], однако, отмечается, что при учете более сложных процессов взаимодействия электрона с фононами процессы, дающие вклад в уравнение (31), являются приближенно упругими. Поэтому время релаксации можно ввести и при низких температурах, что, согласно [18], дает

$$
\begin{array}{ll}
\mu=\frac{3 e}{2 m \alpha \omega_{0}} e^{\nu}, & \nu \gg 1, \\
\mu=\frac{2^{2} e}{3 \pi^{1 / 2} m \alpha \omega_{0}} \nu^{1 / 2}, & \nu \ll 1,
\end{array}
$$

оценки точности формул в [18] отсутствуют. Эти выражения можно считать стандартными результатами, вытекающими в модели Фрёлиха из кинетического уравнения типа Больцмана, для электрона в пределе слабого электрон-фононного взаимодействия $(\alpha \rightarrow 0)$. Приведенное в книге [13] выражение для подвижности при $\nu \ll 1$ совпадает с (75), а при $\nu \gg 1$ отличается числовым множителем. Интересно, что проанализированное нами приближение одного полинома, которое неверно отражает роль в теории полярона распределения Максвелла, дает результат (70), мало отличающийся от (75): при $\nu \gg 1$ в обоих подходах $\mu$ экспоненциально велико и предэкспоненциальные множители мало существенны, а при $\nu \ll 1$ отличие только в числовом множителе.

4) Заметим, что при выборе пробной функции в виде $n$ первых слагаемых в рядах (51) этот принцип дает тот же результат, что использованное выше приближение $n$ полиномов. 
В монографии [2] предложен простой вывод уравнения, полученного в работе [8] для вычисления подвижности полярона. Кинетическое уравнение (31) в стационарном случае умножено на импульс и проинтегрировано по нему. В итоговом соотношении $\Phi Р$ электрона заменена на распределение Максвелла $w_{p-m u}\left(\beta_{0}\right)$ с оговоркой, касающейся физической адекватности такой $\Phi$ Р. В результате получено уравнение

$$
e n E_{l}=\int d^{3} p d^{3} p^{\prime} p_{l} M\left(p, p^{\prime}\right) w_{p^{\prime}-m u}\left(\beta_{0}\right),
$$

которое при $T_{0} \rightarrow 0$ совпадает с уравнением Фейнмана [8]. Предполагая, как и выше, что $u_{l}$ и $E_{l}$ имеют один порядок малости, с учетом определений (44), (49) формы $\left\{A_{p}, B_{p}\right\}$ получим выражение для подвижности электрона

$$
\mu=\frac{3 e}{\beta_{0}\left\{p_{l}, p_{l}\right\}},
$$

которое совпадает с результатом приближения одного полинома (63). Это естественно, так как согласно сказанному выше член первого порядка в разложении распределения Максвелла по скорости совпадает с ФР стационарного состояния системы только в этом приближении.

Полученные в предыдущем разделе результаты, уточняющие приближение одного полинома, будут проанализированы в отдельной работе.

\section{4. ЗАКЛЮЧЕНИЕ}

Показано, что проблемы теории полярона можно эффективно исследовать с помощью функциональной гипотезы Боголюбова, лежащей в основе его метода сокращенного описания неравновесных состояний.

В приближении малого электрон-фононного взаимодействия получено кинетическое уравнение для произвольных неоднородных состояний электрона в полярном кристалле при наличии сильного электрического поля. Задача решена на основе метода сокращенного описания неравновесных состояний с точностью до кубических вкладов по взаимодействию включительно.

На основе обобщения метода Чепмена-Энскога, понимаемого как частный случай метода сокращенного описания, построена теория релаксации скорости и температуры поляронного газа малой плотности. Установлено, что неравновесная одночастичная функция распределения газа отличается от распределения Максвелла уже в линейном приближении по малым разностям скоростей и температур электронной и фононной подсистем. Вычислены соответствующие времена релаксации и подвижность полярона. Полученные результаты конкретизированы в простейшем приближении одного полинома, и произведено сравнение с имеющимися в литературе данными о подвижности. Предложенный метод позволяет изучить нелинейные эффекты в релаксационных явлениях.

Благодарности. Работа была поддержана Государственным фондом фундаментальных исследований Украины (проект № 25.2/102). 


\section{Список литературы}

[1] Н.Н. Боголюбов, Проблемы динамической теории в статистической физике, Гостехиздат, М., Л., 1946.

[2] Н.Н. Боголюбов, Н. Н. Боголюбов (мл.), Аспекты теории полярона, Физматлит, М., 2004; Н. Н. Боголюбов, Собр. научных трудов, т. 7: Введение в квантовую статистическую механику. Аспекты теории полярона, ред. А. Д. Суханов, Наука, М., 2007.

[3] L. Landau, Physikal. Z. UdSSR, 3 (1933), 664-665.

[4] H. Fröhlich, Adv. Phys., 3:11 (1954), 325-361.

[5] А. С. Давыдов, Теория твердого тела, Наука, М., 1976.

[6] А.И. Ахиезер, С. В. Пелетминский, Методъ статистической физики, Наука, М., 1977.

[7] А. И. Соколовский, Укр. физ. журн., 45:4-5 (2000), 548-553.

[8] K. K. Thornber, R. P. Feynman, Phys. Rev. B, 1:10 (1970), 4099-4114.

[9] С. А. Соколовский, И. М. Черненко, Вестник Днепропетровского ун-та. Сер. Физика. Радиоэлектроника, 10 (2003), 152-159.

[10] Дж. Ферцигер, Г. Капер, Математическая теория прочессов переноса в газах, ред. Д. Н. Зубарев, А. Г. Башкиров, Мир, М., 1976.

[11] Л. Д. Ландау, ЖЭТФ, 7 (1937), 203-209.

[12] А.Ф. Александров, Л. С. Богданкевич, А. А. Рухадзе, Основы электродинамики плазмъ, Высшая школа, М., 1988.

[13] Ю. А. Фирсов (ред.), Поляроны, Наука, М., 1975.

[14] S. A. Sokolovsky, "Kinetic equation for electron in polar crystal in the presence of strong electric field", Proc. of 13th International Conference on Mathematical Methods in Electromagnetic Theory (Kyiv, Ukraine, September 6-8, 2010), Wiley-IEEE Press, New York, 2010, 1-4.

[15] И.С. Градштейн, И.М. Рыжик, Таблицы интегралов, сумм, рядов и произведений, Физматлит, М., 1971.

[16] Дж. Займан, Электроны и фононы. Теория явлений переноса в твердых телах, ИЛ, М., 1962.

[17] С. А. Соколовский, А. И. Соколовский, И. М. Черненко, Вестник Харъковского национ. ун-та. Сер. физ. "Ядра, частицы, поля", 642:3(25) (2004), 84-88.

[18] А.И. Ансельм, Введение в теорию полупроводников, Наука, М., Л., 1978.

Поступила в редакцию 16.10.2010, после доработки 16.11.2010 